EUROPEAN JOURNAL OF PURE AND APPLIED MATHEMATICS

Vol. 14, No. 4, 2021, 1266-1274

ISSN 1307-5543 - ejpam.com

Published by New York Business Global

\title{
A New Technique to Speed Up Group Methods for Solving Hyperbolic Telegraph Equations
}

\author{
Abdulkafi Mohammed Saeed \\ Department of Mathematics, College of Science, Qassim University, Buraydah, \\ Kingdom of Saudi Arabia
}

\begin{abstract}
Numerous methods have been introduced in the literature for numerical solution of two-dimensional hyperbolic telegraph equations. Improved techniques using explicit group methods derived from the standard and skewed (rotated) finite difference operators have been developed over the last few years in solving the linear systems that arise from the discretization of the several types of partial differential equations. The preconditioning strategies play a vital role in accelerating the convergence rates of these group iterative methods. In this paper, we present a preliminary study of the formulation of new preconditioned scheme based on explicit group relaxation methods for the difference solution of the telegraph equations. The efficient and robustness of these new formulations over the existing explicit group schemes demonstrated through numerical experiments.
\end{abstract}

2020 Mathematics Subject Classifications: 35B30, 35L10, 35L20, 65M06, 65N06

Key Words and Phrases: Preconditioned technique, Finite difference method, Group iterative method, Telegraph Equations

\section{Introduction}

The group methods depend on rotated finite difference operator were shown to require less execution time requirements than the common point iterative methods based on the centered difference approximations for solving partial differential equations (PDEs) $[1,12-$ 14, 20, 21]. In addition, the methods of the meshless local weak-strong forms combined with the meshless local Petrov-Galerkin are used to solve 2D linear hyperbolic equation by Dehghan and Ghesmati [6]. Besides, the operator splitting method and the spectral Galerkin method have been developed and applied for solving two dimensional hyperbolic equation $[8,10]$. It is well known that preconditioners play a vital role in accelerating the convergence rates of iterative methods, several preconditioned strategies have been used for improving the convergence rate of the explicit group methods derived from the standard and skewed (rotated) finite difference operators [2-5, 15, 17-19]. Based on the existing preconditioning strategies and by combining several categories of preconditioning

DOI: https://doi.org/10.29020/nybg.ejpam.v14i4.4103

Email address: abdulkafi.ahmed@qu.edu.sa (A.M. Saeed) 
techniques, we propose a new preconditioning matrix in block formulation to suit the structure of the explicit group formula for solving telegraph equations.

In the following sections of this research, we will study and discuss the formulation of two types of explicit group methods which are called Explicit group (EG) method and Explicit decoupled group(EDG) method for solving telegraph equations. These iterative methods depend on standard and rotated point iterative schemes respectively. Furthermore, the improvement of the mentioned group methods using preconditioning strategies will be introduced.

This paper is organised as follows: in section 2, we give a presentation of the formulation of explicit group iterative methods such as EG and EDG for solving telegraph equations. The proposed application of the preconditioner in block formulation to the EG and EDG is given in section 3. In section 4, The numerical examples to confirm the results obtained will be presented. Finally, we report a brief conclusion in Section 5 .

\section{Explicit Group(EG-EDG)Methods}

Consider the telegraph equation defined in the region $\Omega=\{(x, y, t): 0<x, y<1, t>$ $0\}$ of the following form [7]:

$$
\frac{\partial^{2} U}{\partial t^{2}}+2 \alpha(x, y, t) \frac{\partial U}{\partial t}+\beta^{2}(x, y, t)=L(x, y, t) \frac{\partial^{2} U}{\partial x^{2}}+M(x, y, t) \frac{\partial^{2} U}{\partial y^{2}}+F(x, y, t)
$$

where $\alpha(x, y, t)>0, \beta(x, y, t) \geq 0, L(x, y, t)>0, M(x, y, t)>0$. The initial and boundary conditions are given by

$$
\begin{aligned}
& U(x, y, 0)=f_{1}(x, y) ; \quad \frac{\partial U}{\partial t}(x, y, 0)=f_{2}(x, y) ; U(0, y, t)=f_{3}(y, t) ; \\
& U(1, y, t)=f_{4}(y, t) ; \quad U(x, 0, t)=f_{5}(y, t) ; \quad U(x, 1, t)=f_{6}(y, t) .
\end{aligned}
$$

Let $k>0$ and $h>0$ be the time step and space step respectively. We divide the interval $0 \leq x, y \leq 1$ into $(N+1)$ subinterval and the grid points are given by $\left(x_{i}, y_{j}, t_{m}\right)=$ $(i h, j h, m k)$ where $m=1,2,3, \ldots$.

Finite difference discretization of equation (1) using centred difference formula for the second partial derivatives will obtain [9]

$$
\begin{aligned}
& \frac{u_{i, j, m+1}-2 u_{i, j, m}+u_{i, j, m-1}}{\Delta t^{2}}+2 \alpha \frac{u_{i, j, m+1}-u_{i, j, m-1}}{2 \Delta t}=\frac{1}{2}\left[\frac{u_{i-1, j, m+1}-2 u_{i, j, m+1}+u_{i+1, j, m+1}}{\Delta x^{2}}\right. \\
& \left.+\frac{u_{i-1, j, m}-2 u_{i, j, m}+u_{i+1, j, m}}{\Delta x^{2}}\right]+\frac{1}{2}\left[\frac{u_{i, j-1, m+1}-2 u_{i, j, m+1}+u_{i, j+1, m+1}}{\Delta y^{2}}+\right. \\
& \left.\frac{u_{i, j-1, m}-2 u_{i, j, m}+u_{i, j+1, m}}{\Delta y^{2}}\right]-\frac{\beta^{2}}{2}\left(u_{i, j, m+1}+u_{i, j, m}\right)+F_{i, j, m+\frac{1}{2}}
\end{aligned}
$$

where $x=i \Delta x, y=j \Delta y, t=m \Delta t ;(i, j=0,1,2, \ldots, n-1 ; m=0,1,2, \ldots)$. The above equation (2) is called standard point formula and after simplification it can be written as

$$
\begin{aligned}
& -\left(\frac{r^{2}}{2}\right) u_{i-1, j, m+1}+\left(1+a+2 r^{2}+b / 2\right) u_{i, j, m+1}-\left(\frac{r^{2}}{2}\right) u_{i+1, j, m+1}-\left(\frac{r^{2}}{2}\right) u_{i, j-1, m+1} \\
& -\left(\frac{r^{2}}{2}\right) u_{i, j+1, m+1}=\left(\frac{r^{2}}{2}\right) u_{i-1, j, m}+\left(2-2 r^{2}-b / 2\right) u_{i, j, m}+\left(\frac{r^{2}}{2}\right) u_{i+1, j, m} \\
& +\left(\frac{r^{2}}{2}\right) u_{i, j-1, m}+\left(\frac{r^{2}}{2}\right) u_{i, j+1, m}+(a-1) u_{i, j, m-1}+\Delta t^{2} F_{i, j, m+\frac{1}{2}}
\end{aligned}
$$


where $h=\Delta x=\Delta y=\frac{1}{N}, r=\frac{\Delta t}{h}, a=\alpha \Delta t, \quad b=\beta^{2} \Delta t^{2}$. By rotating the x-y axis clockwise $45^{0}$, we obtained the rotated finite difference approximation for telegraph equation (1) as follow

$$
\begin{aligned}
& \frac{u_{i, j, m+1}-2 u_{i, j, m}+u_{i, j, m-1}}{\Delta t^{2}}+2 \alpha \frac{u_{i, j, m+1}-u_{i, j, m-1}}{2 \Delta t}=\frac{1}{4}\left[\frac{u_{i-1, j-1, m+1}-2 u_{i, j, m+1}+u_{i+1, j+1, m+1}}{\Delta x^{2}}\right. \\
& \left.+\frac{u_{i-1, j-1, m}-2 u_{i, j, m}+u_{i+1, j+1, m}}{\Delta x^{2}}\right]+\frac{1}{4}\left[\frac{u_{i-1, j+1, m+1}-2 u_{i, j, m+1}+u_{i+1, j-1, m+1}}{\Delta y^{2}}+\right. \\
& \left.\frac{u_{i-1, j+1, m}-2 u_{i, j, m}+u_{i+1, j-1, m}}{\Delta y^{2}}\right]-\frac{\beta^{2}}{2}\left(u_{i, j, m+1}+u_{i, j, m}\right)+F_{i, j, m+\frac{1}{2}}
\end{aligned}
$$

Similarly, we can simplify equation (4) as the following

$$
\begin{aligned}
& -\left(\frac{r^{2}}{4}\right) u_{i-1, j-1, m+1}+\left(1+a+r^{2}+b / 2\right) u_{i, j, m+1}-\left(\frac{r^{2}}{4}\right) u_{i+1, j+1, m+1}-\left(\frac{r^{2}}{4}\right) u_{i-1, j-1, m+1} \\
& -\left(\frac{r^{2}}{4}\right) u_{i+1, j-1, m+1}=\left(\frac{r^{2}}{2}\right) u_{i-1, j-1, m}+\left(2-r^{2}-b / 2\right) u_{i, j, m}+\left(\frac{r^{2}}{4}\right) u_{i+1, j+1, m} \\
& +\left(\frac{r^{2}}{4}\right) u_{i-1, j+1, m}+\left(\frac{r^{2}}{4}\right) u_{i+, j-1, m}+(a-1) u_{i, j, m-1}+\Delta t^{2} F_{i, j, m+\frac{1}{2}}
\end{aligned}
$$

The formulation of EG method depend on the standard point approximation which was derived from the central finite difference discretisation as equations $(2)$ and $(3)[11,16]$. Applying equation (3) to any group of four points on a discretised solution domain will result in a $4 \times 4$ system of equations as follows:

$$
\left(\begin{array}{cccc}
c_{1} & c_{2} & 0 & c_{2} \\
c_{2} & c_{1} & c_{2} & 0 \\
0 & c_{2} & c_{1} & c_{2} \\
c_{2} & 0 & c_{2} & c_{1}
\end{array}\right)\left(\begin{array}{c}
u_{i, j, m+1} \\
u_{i+1, j, m+1} \\
u_{i+1, j+1, m+1} \\
u_{i, j+1, m+1}
\end{array}\right)=\left(\begin{array}{c}
r h s_{i, j} \\
r h s_{i+1, j} \\
r h s_{i+1, j+1} \\
r h s_{i, j+1}
\end{array}\right)
$$

where $c_{1}=1+a+2 r^{2}+\frac{b}{2}, \quad c_{2}=-\frac{r^{2}}{2}$,

$$
\begin{aligned}
& r h s_{i, j}=\left(\frac{r^{2}}{2}\right)\left[u_{i-1, j, m+1}+u_{i, j-1, m+1}\right]+\left(\frac{r^{2}}{2}\right)\left[u_{i-1, j, m}+u_{i, j-1, m}+u_{i+1, j, m}++u_{i, j+1, m}\right] \\
& +\left(2-2 r^{2}-\frac{b}{2}\right) u_{i, j, m}+(a-1) u_{i, j, m-1}+\Delta t^{2} F_{i, j, m+\frac{1}{2}} \\
& r h s_{i+1, j}=\left(\frac{r^{2}}{2}\right)\left[u_{i+1, j-1, m+1}+u_{i+2, j, m+1}\right]+\left(\frac{r^{2}}{2}\right)\left[u_{i, j, m}+u_{i+1, j-1, m}+u_{i+2, j, m}\right. \\
& \left.+u_{i+1, j+1, m}\right]+\left(2-2 r^{2}-\frac{b}{2}\right) u_{i+1, j, m}+(a-1) u_{i+1, j, m-1}+\Delta t^{2} F_{i+1, j, m+\frac{1}{2}} \\
& r h s_{i+1, j+1}=\left(\frac{r^{2}}{2}\right)\left[u_{i+2, j+1, m+1}+u_{i+1, j+2, m+1}+\left(\frac{r^{2}}{2}\right)\left[u_{i, j+1, m}+u_{i+1, j, m}+u_{i+2, j+1, m}\right.\right. \\
& \left.+u_{i+1, j+2, m}\right]+\left(2-2 r^{2}-\frac{b}{2}\right) u_{i+1, j+1, m}+(a-1) u_{i+1, j+1, m-1}+\Delta t^{2} F_{i+1, j+1, m+\frac{1}{2}}, \\
& r h s_{i, j+1}=\left(\frac{r^{2}}{2}\right)\left[u_{i-1, j+1, m+1}+u_{i, j+2, m+1}\right]+\left(\frac{r^{2}}{2}\right)\left[u_{i-1, j+1, m}+u_{i, j, m}+u_{i+1, j+1, m}\right. \\
& \left.+u_{i, j+2, m}\right]+\left(2-2 r^{2}-\frac{b}{2}\right) u_{i, j+1, m}+(a-1) u_{i, j+1, m-1}+\Delta t^{2} F_{i, j+1, m+\frac{1}{2}}
\end{aligned}
$$

The system of equations (6) can be inverted to the following system

$$
\left(\begin{array}{c}
u_{i, j, m+1} \\
u_{i+1, j, m+1} \\
u_{i+1, j+1, m+1} \\
u_{i, j+1, m+1}
\end{array}\right)=\left(\begin{array}{cccc}
\ell_{1} & \ell_{2} & \ell_{3} & \ell_{2} \\
\ell_{2} & \ell_{1} & \ell_{2} & \ell_{3} \\
\ell_{3} & \ell_{2} & \ell_{1} & \ell_{2} \\
\ell_{2} & \ell_{3} & \ell_{2} & \ell_{1}
\end{array}\right)\left(\begin{array}{c}
r h s_{i, j} \\
r h s_{i+1, j} \\
r h s_{i+1, j+1} \\
r h s_{i, j+1}
\end{array}\right)
$$

where

$$
\begin{aligned}
\ell_{1}= & 2\left(4 a^{2}+4 a b+16 a r^{2}+8 a+16 r^{2}+4+8 r^{2} b+4 b+14 r^{4}+b^{2}\right) /\left(8 a^{3}+12 a^{2} b\right. \\
& +48 a^{2} r^{2}+24 a^{2}+24 a b+88 a r^{4}+96 a r^{2}+48 a r^{2} b+24 a+8+6 a b^{2} \\
& \left.+12 b+12 r^{2} b^{2}+48 r^{2}+b^{3}+88 r^{4}+44 r^{4} b+48 r^{6}+48 r^{2} b+6 b^{2}\right)
\end{aligned}
$$




$$
\begin{aligned}
\ell_{2}= & 2 r^{2} /\left(4 a^{2}+4 a b+16 a r^{2}+8 a+4 b+12 r^{4}+16 r^{2}+8 r^{2} b+4+b^{2}\right) ; \\
\ell_{3}= & 4 r^{4} /\left(8 a^{3}+12 a^{2} b+48 a^{2} r^{2}+24 a^{2}+24 a b+88 a r^{4}+96 a r^{2}\right. \\
& +48 a r^{2} b+24 a+6 a b^{2}+12 r^{2} b^{2}+12 b+8+48 r^{2}+b^{3} \\
& \left.+88 r^{4}+44 r^{4} b+48 r^{6}+48 r^{2} b+6 b^{2}\right) .
\end{aligned}
$$

Similarly, the formulation of EDG method can be done by applying equation (5) to any group of four points of the solution domain will result in a $4 \times 4$ system of equations as follows:

$$
\left(\begin{array}{cccc}
q_{1} & q_{2} & 0 & 0 \\
q_{2} & q_{1} & 0 & 0 \\
0 & 0 & q_{1} & q_{2} \\
0 & 0 & q_{2} & q_{1}
\end{array}\right)\left(\begin{array}{c}
u_{i, j, m+1} \\
u_{i+1, j+1, m+1} \\
u_{i+1, j, m+1} \\
u_{i, j+1, m+1}
\end{array}\right)=\left(\begin{array}{c}
r h s_{i, j} \\
r h s_{i+1, j+1} \\
r h s_{i+1, j} \\
r h s_{i, j+1}
\end{array}\right)
$$

where $q_{1}=1+a+r^{2}+\frac{b}{2}, \quad q_{2}=-\frac{r^{2}}{4}$,

$$
\begin{aligned}
& r h s_{i, j}=\left(\frac{r^{2}}{4}\right)\left[u_{i-1, j-1, m+1}+u_{i+1, j-1, m+1}+u_{i-1, j+1, m+1}\right]+\left(\frac{r^{2}}{4}\right)\left[u_{i-1, j-1, m}+u_{i+1, j-1, m}\right. \\
& \left.+u_{i+1, j+1, m}+u_{i-1, j+1, m}\right]+\left(2-r^{2}-\frac{b}{2}\right) u_{i, j, m}+(a-1) u_{i, j, m-1}+\Delta t^{2} F_{i, j, m+\frac{1}{2}}, \\
& r h s_{i+1, j+1}=\left(\frac{r^{2}}{4}\right)\left[u_{i+2, j, m+1}+u_{i+2, j+2, m+1}+u_{i, j+2, m+1}\right]+\left(\frac{r^{2}}{4}\right)\left[u_{i, j, m}\right. \\
& \left.\quad+u_{i+2, j, m}+u_{i+2, j+2, m}+u_{i, j+2, m}\right]+\left(2-r^{2}-\frac{b}{2}\right) u_{i+1, j+1, m} \\
& \quad+(a-1) u_{i+1, j+1, m-1}+\Delta t^{2} F_{i+1, j+1, m+\frac{1}{2}} \\
& r h s_{i+1, j}=\left(\frac{r^{2}}{4}\right)\left[u_{i, j-1, m+1}+u_{i+2, j-1, m+1}+u_{i+2, j+1, m+1}\right]+\left(\frac{r^{2}}{4}\right)\left[u_{i, j-1, m}\right. \\
& \left.\quad+u_{i+2, j-1, m}+u_{i+2, j+1, m}+u_{i, j+1, m}\right]+\left(2-r^{2}-\frac{b}{2}\right) u_{i+1, j, m} \\
& \quad+(a-1) u_{i+1, j, m-1}+\Delta t^{2} F_{i+1, j, m+\frac{1}{2}} \\
& r h s_{i, j+1}=\left(\frac{r^{2}}{4}\right)\left[u_{i-1, j, m+1}+u_{i+1, j+2, m+1}+u_{i-1, j+2, m+1}\right]+\left(\frac{r^{2}}{4}\right)\left[u_{i-1, j, m}\right. \\
& \left.\quad+u_{i+1, j, m}+u_{i+1, j+2, m}+u_{i-1, j+2, m}\right]+\left(2-r^{2}-\frac{b}{2}\right) u_{i, j+1, m} \\
& \quad+(a-1) u_{i, j+1, m-1}+\Delta t^{2} F_{i, j+1, m+\frac{1}{2}} \cdot
\end{aligned}
$$

The system of equations (8) can be written in an explicit decoupled system of $2 \times 2$ equations as follows

$$
\begin{aligned}
& \left(\begin{array}{c}
u_{i, j, m+1} \\
u_{i+1, j+1, m+1}
\end{array}\right)=\frac{1}{L}\left(\begin{array}{ll}
\xi_{1} & \xi_{2} \\
\xi_{2} & \xi_{1}
\end{array}\right)\left(\begin{array}{c}
r h s_{i, j} \\
r h s_{i+1, j+1}
\end{array}\right) \\
& \text { and } \\
& \left(\begin{array}{l}
u_{i+1, j, m+1} \\
u_{i, j+1, m+1}
\end{array}\right)=\frac{1}{L}\left(\begin{array}{ll}
\xi_{1} & \xi_{2} \\
\xi_{2} & \xi_{1}
\end{array}\right)\left(\begin{array}{l}
r h s_{i+1, j} \\
r h s_{i, j+1}
\end{array}\right)
\end{aligned}
$$

where $L=16+32 a+32 r^{2}+16 b+32 a r^{2}+16 a^{2}+16 a b+15 r^{4}+16 r^{2} b+4 b^{2}$; $\xi_{1}=8\left(2+2 a+2 r^{2}+b\right) ; \quad \xi_{2}=4 r^{2}$.

In the EDG method, the grid points are gathered into groups which can consists of only 2 grid points. Each value for $\mathrm{u}$ of every grid point is approximated by the rotated point formula. These values are calculated with a sequence from left to right and then upwards. Hence, the iteration over the solution domain is only carried out on half the mesh points. Once convergence is achieved, the solution at the other half of the points is obtained directly once using the standard point difference formula [1] . 


\section{The Proposed Preconditioned Technique}

The convergence rates of the group iterative methods as EG and EDG depend on the spectral properties of the coefficient matrices $[3,11]$. A preconditioner is a matrix that transforms the resulted system of these methods into one that is equivalent in the sense that it has the same solution, but that has more favourable spectral properties. An ongoing research in this area lies in the formulation of suitable preconditioners which can improve the convergence rates of iterative method $[5,13,14]$. Dramatic improvements are possible, but the difficulty is to construct the suitable preconditioner.

Usually the systems (7) and (9) resulted from EG and EDG methods respectively are large and sparse. By using the following preconditioner matrix

$$
\Psi=\left(\begin{array}{cccc}
q_{1} & 0 & 0 & 0 \\
0 & q_{1} & 0 & 0 \\
0 & 0 & 0 & c_{2} \\
0 & 0 & c_{2} & 0
\end{array}\right)
$$

where $c_{2}$ and $q_{1}$ defined as equations (6) and (8) respectively to both EG and EDG, we will obtain new preconditioned systems. The process of obtaining the new preconditioned system depend on the structure of the coefficient matrix of the target system involves multiplying this matrix $\Psi$ by the original system of the mentioned iterative methods to produce coefficients matrix with a spectral radius less than the spectral radius of the coefficients matrix of the original system.

By applying preconditioner matrix to any group of four points of EG scheme on a discretised solution domain will result in a $4 \times 4$ system of equations as

$$
\left(\begin{array}{cccc}
q_{1} c_{1} & q_{1} c_{2} & 0 & q_{1} c_{2} \\
q_{1} c_{2} & q_{1} c_{1} & q_{1} c_{2} & 0 \\
c_{2}^{2} & 0 & c_{2}^{2} & c_{1} c_{2} \\
0 & c_{2}^{2} & c_{1} c_{2} & c_{2}^{2}
\end{array}\right)\left(\begin{array}{c}
u_{i, j, m+1} \\
u_{i+1, j, m+1} \\
u_{i+1, j+1, m+1} \\
u_{i, j+1, m+1}
\end{array}\right)=\left(\begin{array}{c}
r q_{1} h s_{i, j} \\
r q_{1} h s_{i+1, j} \\
r h c_{2} s_{i, j+1} \\
r h c_{2} s_{i+1, j+1}
\end{array}\right)
$$

The resulted system is called preconditioned EG (PEG) .

By using the same preconditioner matrix and preconditioning process to any group of four points of EDG scheme on a discretised solution domain, we can write the $4 \times 4$ preconditioned EDG (PEDG) system as follows

$$
\left(\begin{array}{cccc}
q_{1}^{2} & q_{1} q_{2} & 0 & 0 \\
q_{1} q_{2} & q_{1}^{2} & 0 & 0 \\
0 & 0 & c_{2} q_{2} & c_{2} q_{1} \\
0 & 0 & c_{2} q_{1} & c_{2} q_{2}
\end{array}\right)\left(\begin{array}{c}
u_{i, j, m+1} \\
u_{i+1, j+1, m+1} \\
u_{i+1, j, m+1} \\
u_{i, j+1, m+1}
\end{array}\right)=\left(\begin{array}{c}
r h s_{i, j} \\
r h s_{i+1, j+1} \\
r h s_{i+1, j} \\
r h s_{i, j+1}
\end{array}\right)
$$

In the following section, we will discuss the efficiency of the above proposed preconditioned system for solving the telegraph equations. 


\section{Numerical Results}

In this section, we check the applicability and effectiveness of the proposed preconditioned group iterative methods in solving problems of telegraph equations. For the purpose of comparison, we use a tolerance of as the termination criteria with the convergence criteria norm. The computer processing unit is Intel(R) Core(TM) i7-7500U with memory of $8 \mathrm{~Gb}$ and the software used to implement and generate the results was Developer $\mathrm{C}++$ Version 4.9.9.2.

All the four methods (EG, EDG, PEG and PEDG) described in sections 2 and 3 were applied to the model problem (1) with $\alpha=\beta=1$ and $L=M=1$ and the initial and boundary conditions are given by

$$
\begin{aligned}
& u(x, y, 0)=\sin (x) \sin (y), \quad u_{t}(x, y, 0)=-\sin (x) \sin (y), \\
& u(0, y, t)=u(x, 0, t)=0, \quad u(1, y, t)=e^{-t} \sin (1) \sin (y), \\
& u(x, 1, t)=e^{-t} \sin (x) \sin (1),
\end{aligned}
$$

with $F(x, y, t)=2 e^{-t} \sin (x) \sin (y)$. The exact solution of this model problem is $u(x, y, t)=$ $e^{-t} \sin (x) \sin (y)$.

In addition, all the mentioned four methods were run using several mesh sizes of 20,50, 80, 98 and 118. The results are summarized in Tables 1 and 2 which showed the comparison among the unpreconditioned EG and EDG methods (original systems) and the preconditioned EG and EDG.

Table 1: Comparison of iterations(k)and elapsed time between EG and EDG methods

\begin{tabular}{|c|cccc|cccc|}
\hline & \multicolumn{4}{|c|}{ Unpreconditioned EG } & \multicolumn{4}{c|}{ Unpreconditioned EDG } \\
$h^{-1}$ & $k$ & Max Error & Ave Error & Time & $k$ & Max Error & Ave Error & Time \\
\hline 20 & 3 & $7.1 E-06$ & $7.3 E-07$ & 0.863 & 3 & $6.9 E-06$ & $8.6 E-07$ & 0.721 \\
50 & 4 & $7.7 E-06$ & $8.5 E-07$ & 4.001 & 3 & $7.4 E-06$ & $8.3 E-07$ & 2.533 \\
80 & 4 & $7.6 E-06$ & $8.4 E-07$ & 13.216 & 3 & $7.3 E-06$ & $8.2 E-07$ & 11.415 \\
98 & 4 & $7.6 E-06$ & $8.3 E-07$ & 12.904 & 3 & $7.3 E-06$ & $8.1 E-07$ & 8.654 \\
118 & 5 & $7.7 E-06$ & $7.5 E-07$ & 14.863 & 4 & $7.6 E-06$ & $7.2 E-07$ & 10.481 \\
\hline
\end{tabular}

Table 2: Comparison of iterations(k)and elapsed time between PEG and PEDG methods

\begin{tabular}{|c|cccc|cccc|}
\hline & \multicolumn{4}{|c|}{ Preconditioned EG } & \multicolumn{4}{c|}{ Preconditioned EDG } \\
$h^{-1}$ & $k$ & Max Error & Ave Error & Time & $k$ & Max Error & Ave Error & Time \\
\hline 20 & 2 & $6.5 E-06$ & $6.2 E-07$ & 0.634 & 1 & $6.5 E-06$ & $5.9 E-07$ & 0.501 \\
50 & 2 & $6.8 E-06$ & $7.7 E-07$ & 1.724 & 1 & $6.6 E-06$ & $7.5 E-07$ & 0.875 \\
80 & 3 & $6.4 E-06$ & $7.5 E-07$ & 10.521 & 2 & $6.1 E-06$ & $7.3 E-07$ & 8.011 \\
98 & 3 & $6.6 E-06$ & $7.2 E-07$ & 7.451 & 2 & $6.1 E-06$ & $7.2 E-07$ & 4.661 \\
118 & 4 & $6.7 E-06$ & $6.4 E-07$ & 8.623 & 3 & $6.6 E-06$ & $6.5 E-07$ & 6.032 \\
\hline
\end{tabular}

We can easily observe that the number of iterations and elapsed time significantly 
reduced when using the new preconditioned methods for solving such problem.

In addition, it becomes clear that the most advanced method with regard to the number of iterations and the elapsed time among all the methods mentioned is the PEDG method. The accuracies of the proposed methods are as good as the original group iterative methods but they require lesser computing timings to achieve the results. Figure 1 shown that the proposed method (PEDG) method is the most time-reducing method due to the lower computations. This means that the new technique used has succeeded in improving the group iterative methods for solving telegraph equations.

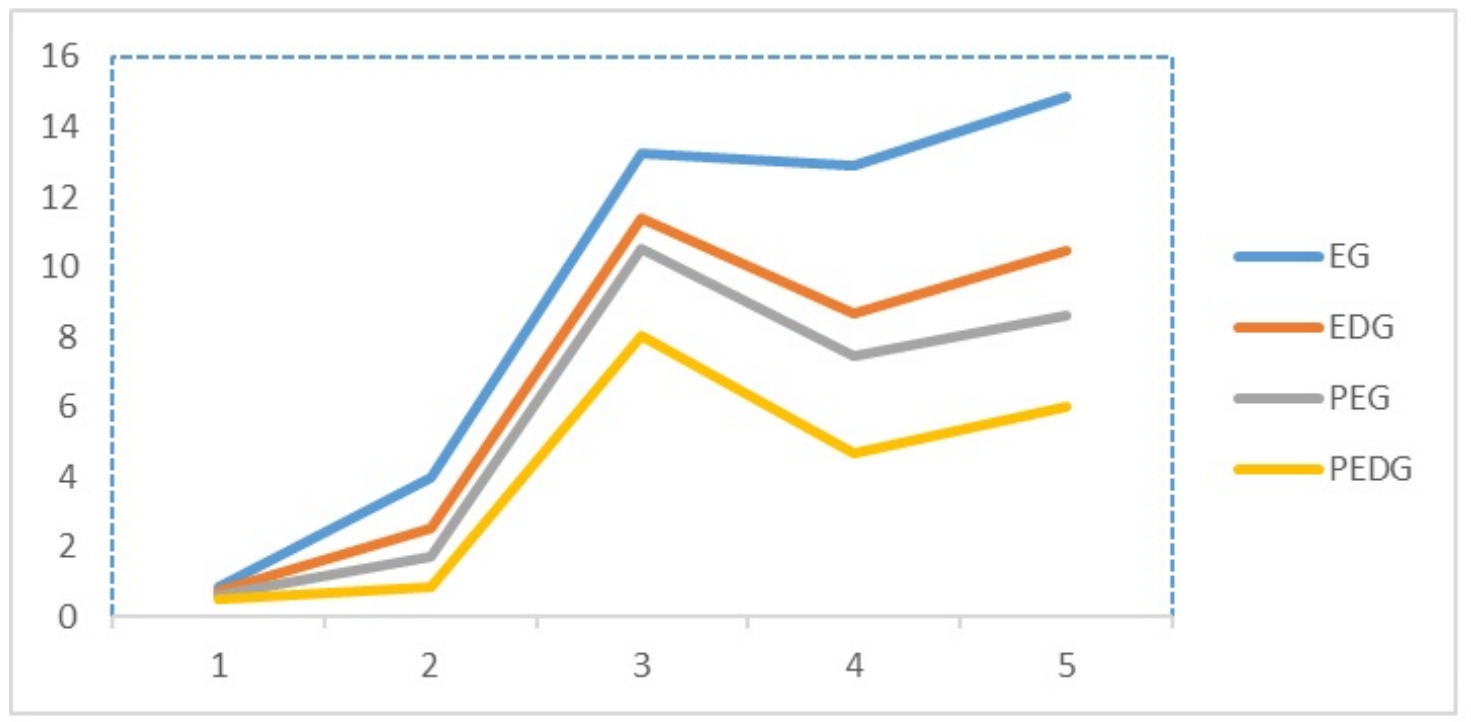

Figure 1: Comparison of elapsed time for all studied methods.

\section{Conclusions}

In this study, we have formulated new preconditioned iterative method based on EG and EDG methods for solving the telegraph initial boundary problems. From observation of all experimental results, it can be concluded that the proposed preconditioned EDG scheme may be a good alternative to solve this type of problems and many other numerical problems. Furthermore, the idea of this preconditioning technique can be extended to solve other types of initial boundary problems which will be reported separately in the future.

\section{Acknowledgements}

The author would like to thank the Department of Mathematics, College of Science and the Deanship of Scientific Research at Qassim University for encouraging scientific 
research.

\section{References}

[1] AR Abdullah. The four point explicit decoupled group (edg) method: A fast poisson solver. International Journal of Computer Mathematics, 38:61-70, 1991.

[2] NHM Ali and DJ Evans. Preconditioned rotated iterative methods in the solution of elliptic pde's. International Journal of Computer Mathematics, 81(9):1163-1174, 2004.

[3] NHM Ali and AM Saeed. Convergence analysis of the preconditioned group splitting methods in boundary value problems. Abstract and Applied Analysis, 2012:1-14, 2012.

[4] NHM Ali and AM Saeed. Preconditioned modified explicit decoupled group for the solution of steady state navier-stokes equation. Applied Mathematics and Information Sciences, 7:1837-1844, 2013.

[5] M Benzi and Z Wang. A relaxed dimensional factorization preconditioner for the incompressible navier-stokes equations. Journal of Computational Physics, 230(34):6185-6202, 2011.

[6] M Dehghan and A Ghesmati. Combination of meshless local weak and strong (mlws) forms to solve the two dimensional hyperbolic telegraph equation. Eng Anal Bound Elem, 34:324-336, 2010.

[7] M Dehghan and A Shokri. A meshless method for numerical solution of a linear hyperbolic equation with variable coefficients in two space dimensions. Numer. Methods Part. Differ. Equ., 25:494-506, 2009.

[8] E Hesameddini and E Asadolahifard. A new spectral galerkin method for solving the two dimensional hyperbolic telegraph equation. Eng Anal Bound Elem, 72:1926-42, 2016.

[9] LM Kew and NHM Ali. Explicit group iterative methods for the solution of telegraph equations. In The 2010 International Conference of Applied and Engineering Mathematics World Congress on Engineering, volume 2010, 2010.

[10] RK Mohanty. An operator splitting method for an unconditionally stable difference scheme for a linear hyperbolic equation with variable coefficients in two space dimensions. Appl Math Comput, 152:799-804, 2004.

[11] M Othman and AR Abdullah. An efficient four points modified explicit group poisson solver. International Journal of Computer Mathematics, 76:203-217, 2002.

[12] AM Saeed. Efficient group iterative method for solving the biharmonic equation. British Journal of Mathematics and Computer Science, 9:237-245, 2015. 
[13] AM Saeed. Improved rotated finite difference method for solving fractional elliptic partial differential equations. American Scientific Research Journal for Engineering, Technology, and Sciences, 25:261-270, 2016.

[14] AM Saeed. A numerical study on medg iterative method for solving nonlinear steady burgers' equation. Global Journal of Engineering Science and Research Management, $25: 32-40,2017$.

[15] AM Saeed. A new version of the accelerated frfd method for solving time-fractional diffusion equations. Journal of Computer Science and Computational Mathematics, $8: 55-57,2018$.

[16] AM Saeed and N AL-harbi. Group splitting with sor/aor methods for solving boundary value problems: A computational comparison. European Journal of Pure and Applied Mathematics, 14:905-914, 2021.

[17] AM Saeed and NHM Ali. On the convergence of the preconditioned group rotated iterative methods in the solution of elliptic pdes. Applied Mathematics and Information Sciences, 5(1):65-73, 2011.

[18] AM Saeed and NHM Ali. Accelerated solution of two dimensional diffusion equation. World Applied Sciences Journal, 7:1906-1912, 2014.

[19] S Xiang and S Zhang. A convergence analysis of block accelerated over-relaxation iterative methods for weak block h-matrices to partition. Linear algebra and its applications, 418:20-32, 2006.

[20] WS Yousif and DJ Evans. Explicit group over-relaxation methods for solving elliptic partial differential equations. Mathematics and Computers in Simulation, 28(6):453466, 1986.

[21] WS Yousif and DJ Evans. Explicit de-coupled group iterative methods and their parallel implementations. Parallel Algorithms and Applications, 7:53-71, 1995. 